\title{
Prevention of falls in hospital 䇺
}

\author{
Authors: Rob Morris ${ }^{\mathrm{A}}$ and Shelagh O'Riordan ${ }^{\mathrm{B}}$
}

Falls among inpatients are the most frequently reported safety incident in NHS hospitals. $30-50 \%$ of falls result in some physical injury and fractures occur in 1-3\%. No fall is harmless, with psychological sequelae leading to lost confidence, delays in functional recovery and prolonged hospitalisation. Yet falls are not true accidents and there is evidence that a coordinated multidisciplinary clinical team approach can reduce their incidence. Identification of multiple underlying risk factors coupled with clear interventions to ameliorate the impact of each has been shown to reduce the incidence of inpatient falls by $20-30 \%$. The implementation of complex multiprofessional interventions is challenging and successful schemes seek to nurture a culture of vigilant safety consciousness in all staff at the clinical interface. Strong leadership and organisational oversight help to combine this cultural evolution with relevant evidence and rigorous measurement of performance in order to improve patient safety. The results of national audit suggest that NHS acute hospitals could do more to reduce the incidence of falls among inpatients.

\section{Introduction}

Falls among hospital inpatients are the most frequently reported safety incident with more than 250,000 recorded annually in England and Wales. ${ }^{1}$ The most recent audit data ${ }^{2}$ show an average of 6.63 falls per 1,000 occupied bed days (OBDs), which equates to more than 1,700 falls every year in an 800 -bed general hospital at current bed occupancy rates. $30-50 \%$ of falls result in some physical injury and fractures occur in $1-3 \% .^{3}$ Even without such injuries, harm to patients, carers and staff frequently manifest through associated psychological distress, fear of further falls, prolonged hospital stays, complaints, litigation, guilt and dissatisfaction. Yet the majority of falls are not true accidents and are not an inevitable consequence of ageing; they are the result of a toxic conspiracy of multiple intrinsic (related to the individual) and extrinsic (environmental influences) risk factors brought to interplay through the riskiness of a person's behaviours. Quite reasonably, the encouragement of mobility is an essential feature of recovery from illness in hospital but poses an obvious

Authors: ${ }^{\text {A }}$ consultant geriatrician and Pathway lead clinician for older people, Nottingham University Hospitals NHS Trust, Nottingham, UK; ${ }^{B}$ consultant geriatrician, Kent Community Health NHS Trust, Ashford, UK dilemma for clinical staff. The unfamiliar hospital setting coupled with concomitant acute physical and/or mental illness serve to amplify the risk of falls for hospitalised older people.

\section{Risk factors for falls in hospital}

The most consistently identified risk factors for falls in hospitalised patients are not dissimilar to those observed in community studies: advanced age ( $>85$ years), male sex, a recent fall, gait instability, agitation and/or confusion, new urinary incontinence or frequency, adverse drug reactions (particularly associated with psychotropic drugs) and neurocardiovascular instability (most notably orthostatic hypotension). ${ }^{4}$ Combinations of risk factors have been used to develop falls risk scores in the hope that prevention efforts can be focused on relatively small numbers of patients at high risk of falling. However, the most widely tested instruments are not sufficiently sensitive to be useful. Current guidance from the National Institute for Health and Care Excellence recommends that all inpatients over the age of 65 and those between 50 and 64 years who have been identified as being at higher risk of falling should be regarded as the population at risk. ${ }^{5}$

\section{Key points}

Falls among hospital inpatients are the most frequently reported safety incident in hospital

Not all falls are preventable but neither are they inevitable: 20$30 \%$ of falls can be prevented by assessing risks and intervening to reduce these risks.

The available fall risk screening tools are too insensitive to be helpful in preventing inpatient falls

We should encourage a culture of vigilant safety consciousness through continuous feedback and learning from adverse events

Preventing falls in hospital is everyone's business; doctors of all grades and disciplines have an important role to play in preventing the harm resulting from inpatient falls

KEYWORDS: Acute hospital, falls, multifactorial falls risk assessments, patient safety, prevention 


\section{Can falls in hospital be prevented?}

Evidence to guide effective falls prevention in hospital is limited with most intervention studies restricted to simple 'before and after' or cluster randomisation designs. ${ }^{5} \mathrm{~A}$ comprehensive review is available $e^{3}$ but in summary: single interventions such as 'high risk' wristbands and bed signage, medication review, urinalysis and routine prescription of vitamin D are not generally successful in reducing falls or fall rates. Although conceptually attractive, the use of movement alarms (bed or chair alarms) has not been successful in reducing fall rates. ${ }^{6}$ In common with community-based trials, more promising results are seen when the complex multiple components of risk are accounted for in assessment and managed through relevant interventions. Systematic reviews have consistently suggested that multifactorial assessments linked to appropriate interventions may reduce falls in hospital by $20-30 \% .^{7-9}$ The optimal combination of components is not, as yet, clearly defined but the assessment of mobility, toileting and continence needs, medication review, vision, confusion (dementia and delirium) and orthostatic blood pressure are important. Similarly, the provision of mobility aids (sticks and walking frames), minimising clutter, clear (pictorial) signage coupled with attention to appropriate footwear, spectacles and hearing aids are simple ways of reducing the potential impact of environmental risk factors.

\section{National Audit of Inpatient Falls}

In 2015, over $90 \%$ of all eligible trusts in England and Wales answered organisational questions about their falls prevention services. Each audited 30 consecutive patients (5,000 in total) to determine whether any or all of those risk factors for falls discussed above were identified and mitigated with appropriate interventions. Fig 1 displays the key results, which show considerable room for improvement. It was also apparent through correlation with organisational level data, that there were significant disparities between written policy and practical implementation thereof at the clinical front line.

\section{Implementing fall prevention processes in NHS hospitals today}

The implementation of complex, multiprofessional interventions is challenging, particularly in the context of the various care settings and patient populations within a general hospital. In contrast to other patient safety themes, such as infection prevention and control, there is little evidence to support a peripatetic approach through the development of specific falls prevention teams. ${ }^{3}$ However, a systematic review suggests a number of key quality improvement themes are important features of successful schemes. ${ }^{9}$ The engagement of front-line staff is critical and requires effective leadership at both ward and board level. Multiprofessional education and training helps to invalidate any prevailing nihilism and promote positive attitudes with respect to falls prevention. Doctors of all grades and specialties have important roles to play in reducing harmful events throughout the acute hospital because almost $70 \%$ of emergency bed days are attributable to patients over 65 years. ${ }^{10}$ Leadership by example at the clinical interface through review of culprit medications (most notably antihypertensive agents and psychotropic drugs), prevention and management of delirium and simple interventions, such as visual assessment, can be done by all physicians. Doctors can also contribute to patient safety through productive engagement in the investigation of falls associated with serious harm by root cause analysis as advised by the Care Quality Commission. ${ }^{11}$ The Care Quality Commission also suggests alternative and less time-consuming methods for managing and learning from all other types of incidents and deliberately moving away from the less informative debate centred on events being avoidable or unavoidable. Continuous learning and improvement, with the involvement of patients and families wherever possible, derives from a clear understanding of the causes of falls rather than the simple identification of omissions in care delivery. At organisational level, the dissemination of learning and oversight of performance by a steering group - as recommended in NICE guidance ${ }^{5}-$ helps to combine culture change and evidence with rigorous measurement of performance.

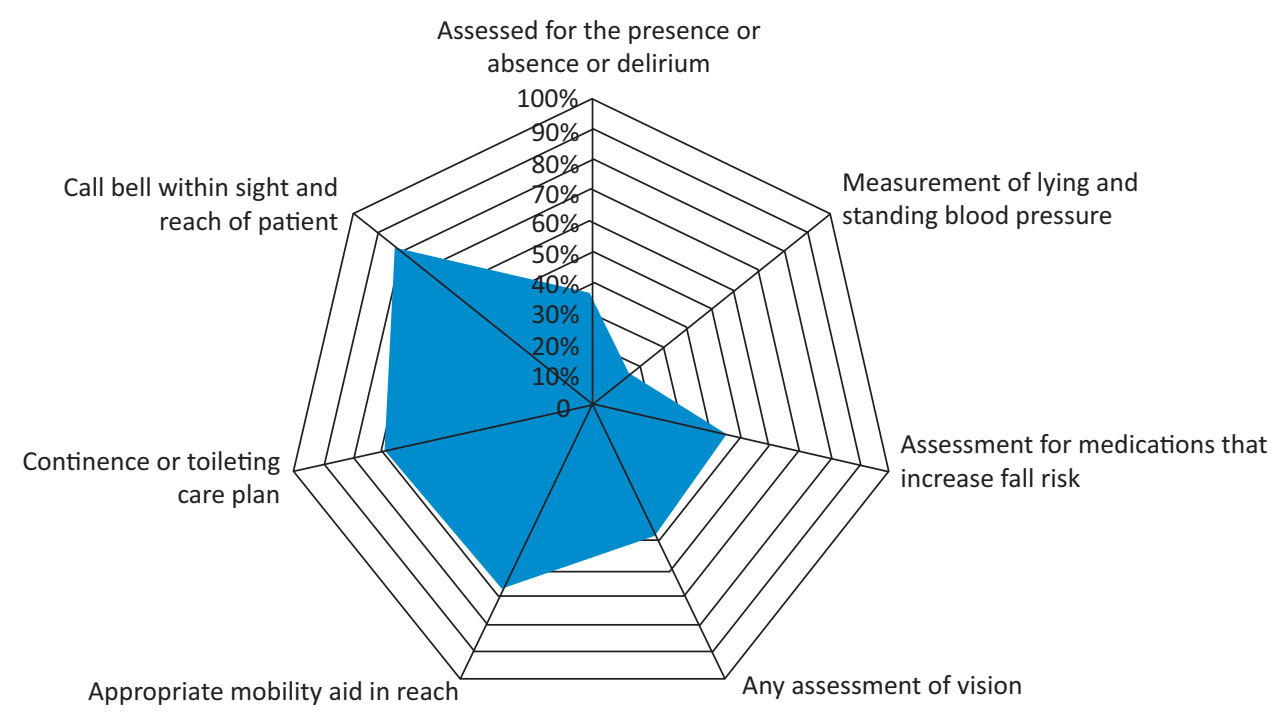

Fig 1. Key indicators for the prevention of falls. Data from the National Audit of Inpatient Falls. ${ }^{2}$ 
There is increasing evidence that nurturing a safety conscious culture within clinical teams can reduce falls as well as other harmful events. An example of this is the use of 'safety huddles', which are short multidisciplinary team briefings that describe the current status of each patient and attempt to identify clinical and non-clinical opportunities to improve patient care and safety. The introduction of safety huddles has been associated with a reduction in falls in some hospitals. ${ }^{12}$ Other examples would be the use of 'intentional rounding' and improved handover systems to include the discussion of patients at high risk of falls and interventions underway or required. All of these methods involve a degree of 'bottomup' implementation and the empowerment of staff to trial interventions and adjust their application until successful. The inclusive co-design and ongoing development of interventions at the clinical interface nurtures ownership and, with sensitive real-time feedback on performance, facilitates the potential for continuous improvement. The alternative use of prescribed care bundles, such as the Royal College of Physicians' FallSafe ${ }^{13}$ programme has shown promising results. The National Audit of Inpatient Falls has also stimulated the development of new tools to aid visual assessment ${ }^{14}$ and the measurement of orthostatic blood pressure ${ }^{15}$ at the bedside.

\section{Conclusions}

Falls are the most commonly reported patient safety incident in the acute hospital setting. Falls can result in death or severe injury, including fractures; no fall can be regarded as entirely harmless because they are also associated with fear of further falls, delayed functional recovery and increased length of hospital stay. There are multiple risk factors for falling in hospital but no screening instrument has proven sufficiently sensitive to be clinically useful. It is recommended, therefore, that all hospitalised patients over the age of 65 years (and those over 54 years judged to have specific risks) should have a multifactorial falls risk assessment. Identifying risks and providing effective interventions where possible can reduce falls by $20-30 \%$. Building safety consciousness into clinical teams and embedding routine vigilance are emerging features of successful harm reduction schemes. However, results from the National Audit of Inpatient Falls showed that many trusts are missing the opportunity to reduce falls in hospital.

Falls prevention in hospital is everyone's business, from the trust board to all staff at the clinical interface. The best practices seem to include empowering multidisciplinary teams to test and refine interventions intended to mitigate risk factors shown to be important through investigative governance systems, which were designed to elucidate the causes of inpatient falls.

\section{Conflicts of interest}

The authors have no conflicts of interest to declare.

\section{References}

1 Agency National Patient Safety. Slips trips and falls data update: from acute and community hospitals and mental health units in England and Wales. London: NPSA, 2010. www.nrls.npsa. nhs.uk/resources/patient-safety-topics/patient-accidentsfalls/?entryid45=74567 [Accessed 22 May 2017].

2 Royal College of Physicians. National Audit of Inpatient Falls: audit report 2015. London: RCP, 2015.

3 Oliver D, Healey F, Haines T. Preventing falls and fall-related injuries in hospitals. Clin Geriatr Med 2010;26:645-92.

4 Oliver D, Daly F, Martin FC. Risk factors and risk assessment tools for falls in hospital inpatients. A systematic review. Age Ageing 2004;33:122-30.

5 National Institute for Health and Care Excellence. Falls in older people: assessing risk and prevention. NICE clinical guideline No 161. Manchester: NICE, 2013.

6 Sahota O, Drummond A, Kendrick D et al. REFINE (REducing Falls in In-patieNt Elderly) using bed and bedside chair pressure sensors linked to radio-pagers in acute hospital care: a randomised controlled trial. Age Ageing 2014;43:247-53.

7 Cameron ID, Gillespie LD, Robertson MC et al. Interventions for preventing falls in older people in care facilities and hospitals. Cochrane Database Syst Rev 2013;(12):CD005465.

8 Oliver D, Connelly JB, Victor CR et al. Strategies to prevent falls and fractures in hospitals and care homes and effect of cognitive impairment: systematic review and meta-analyses. $\mathrm{Br} \mathrm{Med} \mathrm{J}$ 2007;334:82-7.

9 Miake-Lye IM, Hempel S, Ganz DA, Shekelle PG. Inpatient fall prevention programs as a patient safety strategy a systematic review. Ann Intern Med 2013;158:390-6.

10 Imison C, Poteliakhoff E, Thompson J. Older people and emergency bed use. Exploring variation. London: The King's Fund, 2012.

11 Care Quality Commission. Learning from serious incidents in NHS acute hospitals: a review of the quality of investigation reports. Newcastle upon Tyne: CQC, 2016.

12 Cracknell A, Lovatt A, Winfield A et al. Huddle up for safer healthcare: how frontline teams can work together to improve patient safety. Future Hospital Journal 2016;3(Suppl 2):s31.

13 Healey F, Lowe D, Darowski A et al. Falls prevention in hospitals and mental health units: an extended evaluation of the FallSafe quality improvement project. Age Ageing 2014;43:484-91.

14 Windsor J, Dix A. A bedside tool to assess eyesight in hospital patients at risk of falls. Nursing Times 2017;113:22-4.

15 Royal College of Physicians. Measurement of lying and standing blood pressure: a brief guide for clinical staff. London: RCP, 2017. www.rcplondon.ac.uk/projects/outputs/measurement-lying-andstanding-blood-pressure-brief-guide-clinical-staff [Accessed 22 May 2017].

Address for correspondence: Dr Rob Morris, Health Care for Older People, Queen's Medical Centre, Derby Road, Nottingham NG7 2UH, UK.

Email: Rob.Morris@nuh.nhs.uk 